\title{
Delirium in the Intensive Care Unit: Incidence, Risk Factors, and Impact on Outcome
}

\author{
Nejla Tilouche ${ }^{1,2}$, Mohamed Fekih Hassen ${ }^{1,2}$, Habiba Ben Sik Ali1,2, Oussamma Jaoued ${ }^{1,2}$, Rim Gharbi ${ }^{1,2}$, S. Souheil El Atrous ${ }^{1,2}$ \\ ${ }^{1}$ Intensive Care Unit, University Hospital Taher Sfar Mahdia 5100, Tunisia, ${ }^{2}$ University of Monastir, Research Laboratory, LR 12 SP15
}

\section{Abstract}

Background: The incidence and risk factors for delirium vary among studies. Objective: We aimed to determine the incidence, risk factors, and impact on outcome of delirium in a medical Intensive Care Unit (ICU) in Tunisia using a prospective observational study. Patients: All consecutive patients admitted to the ICU between May 2012 and April 2013 were included if they were aged more than 18 years and had an ICU stay of more than $24 \mathrm{~h}$. Patients who had a cardiac arrest or have a history of dementia or psychosis were excluded. Patients eligible for the study were evaluated by the medical staff to detect delirium using the CAM-ICU. Results: A total of 206 patients were included, 167 did not present delirium and $39(19 \%)$ were analyzed for delirium. Delirious patients had a significantly longer duration of mechanical ventilation (10 days[6-20] vs. 2 days[0-7]) respectively and length of stay in ICU (21.5 days [10.5-32.5] vs. 8 days [5-13]), with no impact on mortality. Delirium was associated with high incidence of unintentional removal of catheters $(39 \% \mathrm{vs} .9 \% ; P<0.0001)$, endotracheal tubes $(18 \%$ vs. $1 \% ; P<0.0001)$, and urinary catheters $(28 \%$ vs. $2 \%, P<0.0001)$. In multivariable risk regression analysis, age (odds ratio [OR] $=4.1,95 \%$ confidence interval $[\mathrm{CI}]: 1.39-12.21 ; P=0.01)$, hypertension $(\mathrm{OR}=3.3,95 \% \mathrm{CI}: 1.31-8.13 ; P=0.011), \mathrm{COPD}(\mathrm{OR}=3.5,95 \% \mathrm{CI}: 1.47-8.59$; $P=0.005)$, steroids $(\mathrm{OR}=2.8,95 \% \mathrm{CI}: 1.05-7.28 ; P=0.038)$, and sedation $(\mathrm{OR}=5.4,95 \% \mathrm{CI}: 2.08-13.9 ; P<0.0001)$ were independent risk factors for delirium. We did not find a relationship between delirium and mortality. Conclusion: Delirium is frequent in the ICU and is associated with poor outcome. Several risk factors for delirium are linked to intensive care environment.

Keywords: Delirium, Intensive Care Unit, incidence, mortality, risk factors

\section{INTRODUCTION}

Delirium is a disturbance of consciousness, cognition, attention, and perception. These symptoms usually have an acute onset and a transient fluctuation along the day. ${ }^{[1]}$

Hyperactive delirium which is a state of great agitation has often been described as the most common subtype observed in clinical practice and can Wbe misdiagnosed as psychotic disorder or an agitated dementia. Hypoactive delirium occurs frequently but has a bad prognosis because of lack of recognition by clinicians. ${ }^{[2]}$

Previous studies reported an incidence of delirium in Intensive Care Unit (ICU) between $11 \%$ and $87 \%{ }^{[3,4]}$ The risk of delirium is particularly high in elderly people, those with preexisting cognitive impairments, ${ }^{[5,6]}$ terminal illnesses, ${ }^{[7]}$ patients undergoing major surgery, ${ }^{[8]}$ and those who are admitted to an ICU. ${ }^{[10-12]}$ Comorbidities, severity of illness, drugs and tobacco withdrawal, ICU conditions, and several treatments are common risk factors for delirium in ICU. ${ }^{[5,9-12]}$

\begin{tabular}{|l|l|}
\hline \multicolumn{3}{c|}{ Access this article online } \\
\hline Quick Response Code: & Website: \\
& www.ijccm.org \\
& \\
&
\end{tabular}

Delirium can result in bad outcome including prolonged mechanical ventilation, longer ICU and hospital length of stay (LOS), higher mortality, long-term cognitive impairment ${ }^{[13-15]}$ and higher cost of care. ${ }^{[16]}$

We believe that incidence and risk factors for delirium may be different in a Tunisian ICU given the type of patients less frequently alcoholic because of religious beliefs; additionally, COPD, known as a risk factor for delirium, represents $>30 \%$ of admissions in our unit. Our practices may also be different from those observed in other ICUs since we do not use a protocol of sedation and we do not allow visits in the patient's bedside.

Address for correspondence: Dr. Mohamed Fekih Hassen, Intensive Care Unit University Hospital Taher Sfar Mahdia 5100, Tunisia. E-mail: mohamed.fekihhassen1@gmail.com Dr. Nejla Tilouche,

Intensive Care Unit University Hospital Taher Sfar Mahdia 5100, Tunisia. E-mail: tilouche.nejla@gmail.com

This is an open access journal, and articles are distributed under the terms of the Creative Commons Attribution-NonCommercial-ShareAlike 4.0 License, which allows others to remix, tweak, and build upon the work non-commercially, as long as appropriate credit is given and the new creations are licensed under the identical terms.

For reprints contact: reprints@medknow.com

How to cite this article: Tilouche N, Hassen MF, Ali HB, Jaoued O, Gharbi R, El Atrous SS. Delirium in the Intensive Care Unit: Incidence, risk factors, and impact on outcome. Indian J Crit Care Med 2018;22:144-9. 
This study aimed to determine the incidence, risk factors, and impact on outcome of delirium in a medical ICU.

\section{Patients}

We conducted a prospective and observational study in the medical ICU of a teaching hospital. Admissions are received from the emergency department and medical wards with an average of 350 admissions per year. Visits are possible through a corridor that overlooks the bedrooms' windows.

All consecutive patients admitted to the ICU between May 2012 and April 2013 were included in the study if they aged $>18$ years and had an ICU stay of $>24 \mathrm{~h}$. Patients with a not to resuscitate decision, who had a cardiac arrest or had a history of dementia or psychosis, were excluded from the study because of the difficulty to assess delirium in this group.

Patients' consent was not warranted given the observational nature of the study.

\section{Methods}

Patients eligible for the study were evaluated in the morning from Monday to Saturday until discharge by the medical staff to detect delirium using the Confusion Assessment Method (CAM)-ICU. [3]

We started the process of evaluation by assessing the sedation level via the Riker Agitation-Sedation Scale (SAS). It is an instrument simple to use; the score varies from 1 to 7: level (1) refers to a patient who cannot respond to voice or physical stimulation and (7) to a combative violent patient. Every patient was daily evaluated until his/her SAS score is $\geq 4$, so he/she can be assessed for delirium using the CAM-ICU.

According to the CAM-ICU, patients had a diagnosis of delirium when an acute onset of mental status change and inattention were accompanied by either disorganized thinking or an altered level of consciousness.

To define the duration of delirium, we daily calculated the Delirium Detection Score (DDS) daily in delirious patients; this score evaluates 5 items: agitation, anxiety, hallucination, orientation, and paroxysmal sweating. ${ }^{[17]}$

A patient still has delirium if his/her DDS level is $\geq 8$.

\section{Data collection}

Risk factors associated with delirium were documented and subdevised into predisposing factors present before ICU admission and precipitating factors occurring during critical illness and which are changeable by preventive or therapeutic intervention.

The information collected at the time of enrollment included patient demographics, comorbidities, smoking status, alcohol, admission diagnosis, Simplified Acute Physiology Score II (SAPSII), laboratory investigations, treatments, duration of mechanical ventilation, ICU LOS, and mortality.

\section{Statistical analysis}

The statistical analyses were performed using SPSS 18.0 (Chicago, IL, USA). The descriptive data are presented as the number and percentage for categorical data and mean \pm standard deviation and median (25-75 percentiles interquartile ranges, IQR) for continuous data according to their distribution. The cohort study is divided into two groups: ever delirium and never delirium. Chi-square test and Fisher's exact probability test were used to detect the difference between groups in the univariate analysis, as appropriate.

The risk factors were analyzed using binary logistic regression. Any variables which had $P<0.20$ after the univariable risk regression and all other potential variables associated with the occurrence of delirium were included for the multivariable risk regression. The level of significance was set at $P<0.05$.

\section{Results}

In a 12-month period, 336 patients were admitted in our ICU and 206 patients were included in the study [Figure 1].

Reasons for exclusion were age $<18$ years $(n=14)$, postcardiac arrest $(n=26)$, LOS $<24 \mathrm{~h}(n=66)$, decision of not to resuscitate $(n=18)$, and dementia or psychosis $(n=6)$.

Patients' baseline characteristics are presented in Table 1. The incidence of delirium was $19 \%$ and the mean DDS score was $10 \pm 2$. Delirium was mixed in $12 \%$, hyperactive in $44 \%$, and hypoactive in $44 \%$. The median onset of delirium was 8 days (IQR 3-13) with a median duration of 3 days (IQR 2-4). Delirious patients were significantly older (71 \pm 9 vs. $61 \pm 17$ years; $P<0.001$ ), had higher SAPS II score $(38 \pm 15$ vs. $30 \pm 15 ; P<0.007)$, higher incidence of hypertension ( $56 \%$ vs. $38 \% ; P=0.032$ ), and COPD (72\% vs. $23 \% ; P<0.001)$.

Respiratory failure was found more frequently in patients who developed delirium (77\% vs. 58\%; $P=0.025)$.

Metabolic disorders such as respiratory acidosis (46\% vs. 17\%; $P<0.01)$ and chronic hypercapnia $(72 \%$ vs. $23 \% ; P<0.0001)$ were significantly higher in delirium group. However,

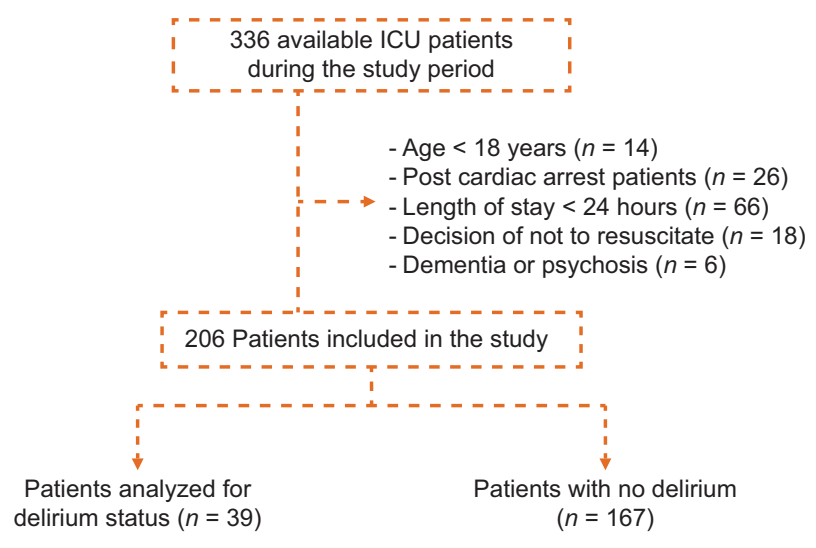

Figure 1: The flow diagram of patients in the study 


\begin{tabular}{|c|c|c|c|}
\hline & $\begin{array}{c}\text { Delirious } \\
\text { patients } \\
(n=39), n(\%)\end{array}$ & $\begin{array}{c}\text { Nondelirious } \\
\text { patients } \\
(n=167), n(\%)\end{array}$ & $P$ \\
\hline Age (years) & $71 \pm 9$ & $61 \pm 17$ & $<0.001$ \\
\hline Gender (male/female) & $26 / 13$ & $88 / 79$ & 0.11 \\
\hline SAPS II & $38 \pm 15$ & $30 \pm 15$ & 0.007 \\
\hline \multicolumn{4}{|l|}{ Comorbidity } \\
\hline Hypertension & $22(56)$ & $63(38)$ & 0.032 \\
\hline Diabetes mellitus & $7(18)$ & $42(25)$ & 0.342 \\
\hline Chronic renal failure & $3(8)$ & $15(9)$ & 0.54 \\
\hline COPD & $28(72)$ & $39(23)$ & $<0.001$ \\
\hline \multicolumn{4}{|l|}{ History } \\
\hline Alcohol & $2(5)$ & $6(4)$ & 0.64 \\
\hline Current smokers & $13(33)$ & $34(20)$ & 0.08 \\
\hline \multicolumn{4}{|l|}{ Reason of ICU admission } \\
\hline Acute respiratory failure & $30(77)$ & $96(58)$ & 0.025 \\
\hline Septic shock & $4(10)$ & $14(8)$ & 0.75 \\
\hline Coma & $1(2.5)$ & $27(16)$ & 0.025 \\
\hline Other & 0 & $15(9)$ & 0.12 \\
\hline
\end{tabular}

COPD: Chronic obstructive pulmonary disease; SAPS II: Simplified Acute Physiology Score II; ICU: Intensive Care Unit

\begin{tabular}{|c|c|c|c|}
\hline & $\begin{array}{c}\text { Nondelirious } \\
\text { patients } \\
(n=167), n(\%)\end{array}$ & $\begin{array}{c}\text { Delirious } \\
\text { patients } \\
(n=39), n(\%)\end{array}$ & $P$ \\
\hline Anemia & $34(20)$ & $10(25.6)$ & 0.51 \\
\hline Hyperthermia & $32(19)$ & $9(26)$ & 0.65 \\
\hline Kidney failure & $18(11)$ & $3(8)$ & 0.77 \\
\hline Hemodiafiltration & $5(3)$ & $1(2.5)$ & 0.97 \\
\hline Hemodialysis & $13(8)$ & $2(5)$ & 0.7416 \\
\hline Liver failure & $14(8)$ & $2(5)$ & 0.7416 \\
\hline Dehydration & $15(9)$ & $1(3)$ & 0.316 \\
\hline \multicolumn{4}{|l|}{ Metabolic disorder } \\
\hline Hyponatremia & $23(14)$ & $0(0)$ & 0.009 \\
\hline Hypokalemia & $22(13)$ & $1(3)$ & 0.285 \\
\hline Hyperkalemia & $9(5)$ & $3(8)$ & 0.702 \\
\hline Hypoglycemia & $2(1)$ & $0(0)$ & 1 \\
\hline Metabolic acidosis & $16(10)$ & $3(8)$ & 0.001 \\
\hline Respiratory acidosis & $29(17)$ & $18(46)$ & 0.00011 \\
\hline Acute hypercapnia & $9(5)$ & $0(0)$ & 0.212 \\
\hline Chronic hypercapnia & $39(23)$ & $28(72)$ & $<0.0001$ \\
\hline Hypoxemia & $12(7)$ & $3(8)$ & 0.021 \\
\hline Sedation & $31(79)$ & $59(35)$ & $<0.001$ \\
\hline \multicolumn{4}{|l|}{ Molecules } \\
\hline Analgesic & $31(79)$ & $58(35)$ & $<0.001$ \\
\hline Midazolam & $31(79)$ & $59(35)$ & $<0.001$ \\
\hline Antipsychotic drugs & $2(5)$ & $9(5)$ & 1 \\
\hline Steroids & $16(41)$ & $37(22)$ & 0.015 \\
\hline Anticonvulsants & $4(10)$ & $11(7)$ & 0.49 \\
\hline Vasopressor & $19(49)$ & $36(22)$ & 0.001 \\
\hline$\beta 2$ agonists & $9(23)$ & $22(13)$ & 0.119 \\
\hline Mechanical ventilation & $36(92)$ & $94(56)$ & $<0.001$ \\
\hline
\end{tabular}

hyponatremia ( $14 \%$ vs. $0 \% ; P=0.009$ ) and metabolic acidosis were more frequently found in nondelirious patients [Table 2].

Patients who received a continuous infusion of midazolam $(79 \%$ vs. $35 \%, P<0.001)$, analgesics $(79 \%$ vs. $35 \%$, $P<0.001)$, steroids ( $41 \%$ vs. $22 \%, P=0.015)$, and vasopressor support ( $49 \%$ vs. $22 \%, P=0.01$ ) were higher in the delirium group [Table 2].

In multivariate risk regression analysis, age (odds ratio $[\mathrm{OR}]=4.1,95 \%$ confidence interval $[\mathrm{CI}]: 1.39-12.21$; $P=0.01)$, hypertension $(\mathrm{OR}=3.3,95 \% \mathrm{CI}: 1.31-8.13$; $P=0.011)$, COPD $(\mathrm{OR}=3.5,95 \% \mathrm{CI}: 1.47-8.59 ; P=0.005)$, steroids $(\mathrm{OR}=2.8,95 \% \mathrm{CI}: 1.05-7.28 ; P=0.038)$, and sedation $(\mathrm{OR}=5.4,95 \% \mathrm{CI}: 2.08-13.90 ; P<0.0001)$ were independent risk factors for delirium [Table 3].

\section{Impact of delirium}

Delirium was associated with high incidence of unintentional removal of catheters (39\% vs. $9 \% ; P<0.0001)$, endotracheal tubes $(18 \%$ vs. $1 \% ; P<0.0001)$, and urinary catheters $(28 \%$ vs. $2 \% ; P<0.0001)$ [Table 4 ].

Delirious patients had significantly longer median ventilation days $(10[6-20]$ vs. $2[0-7] ; P<0.0001)$ and ICU LOS (21.5 [10.5-32.5] vs. $8[5-13] ; P<0.0001)$. Mortality was similar between the two groups $(16 \%$ vs. $15 \% ; P=0.9)$ [Table 4].

\section{Discussion}

The overall incidence of delirium in our study was $19 \%$. Delirium was associated with longer LOS in ICU and longer duration of mechanical ventilation. We did not find a relationship between delirium and mortality. In multivariate risk regression analysis, age, hypertension, COPD, corticosteroids, and sedation were independent risk factors for delirium.

In the present study, the incidence of delirium is in the range reported in the literature.$^{[3,4,18-20]}$ However, a higher incidence was found in many other studies which can be explained by differences in definitions, type of ICU population (medical or surgical), severity of illness, and diagnosis tool used. ${ }^{[21-24]}$

In addition, we did not assess delirium at night, and some patients with hypoactive delirium may not be detected in our study because of the difficulty of diagnosis in ventilated patients. Finally, we excluded patients with decision of not to resuscitate.

The mean DDS score was $10 \pm 2$, and $60 \%$ of patients developed delirium in the $2^{\text {nd }}$ week. This delay in onset of delirium compared to other studies, in which delay is between 1 and 5 days, ${ }^{[10,18]}$ may be related to the high percentage of patients ventilated and deeply sedated during the $1^{\text {st }}$ days. The mean duration of delirium was $3 \pm 1$ days, which matched previous findings. ${ }^{[10,18,21]}$

In this study, hyperactive and hypoactive delirium occurred with the same frequency (44\%), of which mixed subtype was 
the least common. This distribution is different in the literature with a higher frequency of mixed subtype. ${ }^{[25,26]}$

Five risk factors for delirium were identified in our study: the use of steroids and sedation, presence of COPD, hypertension, and age. According to many studies, age is not a predictor of delirium. ${ }^{[27,28]}$ However, Pandharipande et al. found that the probability of delirium increases dramatically after 65 years. ${ }^{[11]}$ In our study, age increased by 4 -fold the risk of delirium although our patients have no known history of dementia. This can be explained by the vulnerability of old patients to metabolic disturbances and hypoxemia in addition to psychological stress in an intensive care environment.

COPD was an important risk factor for delirium in our patients $(\mathrm{OR}=3.5,95 \% \mathrm{CI}: 1.479-8.595 ; P=0.005)$. Similar findings were reported by Shi et al. ${ }^{[29]}$ and Aldemir et al. ${ }^{[12]}$ These patients are particularly exposed to psychological disturbances caused by hypoxemia, hypercapnia, and tobacco withdrawal. Furthermore, patients with COPD are frequently treated with corticosteroids ( $25 \%$ ), which is itself a risk factor for delirium in our study.

Hypertension was also found to be a risk factor for delirium in our study, which is consistent with the results of Dubois et al. and Ouimet et al..$^{[9,10]}$

Hypertension can be associated with a decline in cognitive performance. In fact, the vascular damage exposes hypertensive patients to cerebral hypoperfusion and cerebral hypoxia and makes them at higher risk for delirium when hospitalized in the ICU. ${ }^{[30,31]}$

Table 3: Multivariable analysis of risk factors related to delirium

\begin{tabular}{lccc}
\hline & OR & OR $\mathbf{( 9 5 \% ~ C I )}$ & $\boldsymbol{P}$ \\
\hline Age & 4.1 & $1.399-12.216$ & 0.01 \\
Hypertension & 3.3 & $1.312-8.133$ & 0.011 \\
COPD & 3.5 & $1.479-8.595$ & 0.005 \\
Steroids & 2.8 & $1.057-7.284$ & 0.038 \\
Sedation & 5.4 & $2.088-13,924$ & $<0.0001$ \\
\hline
\end{tabular}

COPD: Chronic obstructive pulmonary disease; OR: Odds ratio;

CI: Confidence interval
Two other alterable risk factors detected in our study are the use of steroids and sedative drugs mainly benzodiazepine and morphine analgesics. The use of sedation increased by 5 -fold the risk of delirium. The absence of sedation protocol can explain this result but sedation, especially benzodiazepine, was implicated in delirium several times. In a study including trauma and postoperative patients, midazolam, the most used in sedation for our patients, increased by $2-3$ folds the risk of delirium; ${ }^{[32]}$ therefore, many studies were conducted to find an alternative for midazolam. Dexmedetomidine seemed to be the most attractive choice. The MENDS trial showed a superiority of dexmedetomidine compared to lorazepam in ventilated patients with more delirium-free days and best sedation target achievement. ${ }^{[33]}$

The increased risk of delirium linked to the use of corticosteroids in our study may be surprising since the pathophysiology of delirium includes a cerebral inflammation as a response to insult, but such complication has been reported several times especially in older persons. ${ }^{[34-36]}$ Agitation correlated to steroids is well known and the reasons remain unidentified; furthermore, no study found a dose relationship.

Like many other reports, we did not find an impact of delirium on ICU survival. ${ }^{[23,37-39]}$ A relationship between delirium and long-term mortality is not excluded. Mortality may be influenced by duration of delirium as reported by Shehabi et al. This result was not searched in the present study. ${ }^{[40]}$

We found an association between LOS and delirium. A meta-analysis including 28 studies reported an ICU LOS significantly longer in patients with delirium (standardized mean difference $=1.38,95 \% \mathrm{CI}: 0.99-1.77 ; P<0.001) .{ }^{[41]}$

In our study, mechanical ventilation was extended by 8 days in delirious patients. Our results are comparable to those of Lat et al. ${ }^{[38]}$ and Salluh et al. ${ }^{[41]}$ the latter found a mean duration of mechanical ventilation of 1.79 days longer in patients with delirium.

Side effects were significantly higher in the delirium group. This result was confirmed by Jaber et al. ${ }^{[37]}$

We must highlight several limitations of our research. First, it is a single ICU study. Second, delirium was not assessed

\begin{tabular}{|c|c|c|c|}
\hline & Delirious patients $(n=39), n(\%)$ & Nondelirious patients ( $n=167), n$ (\%) & $P$ \\
\hline Duration of MV; median (IQR) & $10(6-20)$ & $2(0-7)$ & $<0.0001$ \\
\hline ICU LOS, days; median (IQR) & $21.5(10.5-32.5)$ & $8(5-13)$ & $<0.0001$ \\
\hline Mortality & $6(15)$ & $27(16)$ & 0.9 \\
\hline \multicolumn{4}{|l|}{ Complications } \\
\hline Unintentional tube removal & $7(18)$ & $1(1)$ & $<0.0001$ \\
\hline Unintentional central removal & $3(8)$ & $1(1)$ & 0.02 \\
\hline Perfusion removal & $12(31)$ & $13(8)$ & $<0.0001$ \\
\hline Nasogastric tubes removal & $2(5)$ & $1(1)$ & 0.09 \\
\hline Urinary catheter removal & $11(28)$ & $3(2)$ & $<0.0001$ \\
\hline
\end{tabular}

ICU: Intensive Care Unit; LOS: Length of stay, MV: Mechanical ventilation; IQR: Interquartile range 
at night and we may have missed some cases of delirium. Doses of sedatives and steroids were not recorded, so we cannot determine a dose relationship and whether the absence of protocol is a factor of sedative overuse. Finally, we did not assess long-term outcome to detect persistent cognitive impairment.

The association between delirium and poor outcome is clear. Future studies should focus on therapeutic options in addition to a strategy of early detection of brain dysfunction. We should also evaluate the effect of prevention on the incidence of delirium.

\section{CONCLUSION}

In the present study, we found an incidence of delirium in ICU among ventilated and nonventilated patients of $19 \%$. Age, SAPS, hypertension, COPD, and use of sedation and corticosteroids are important risk factors for delirium. Delirium resulted in longer ICU stay, prolonged mechanical ventilation, and exposed our patients to adverse events. Mortality in the ICU was unchanged by delirium.

\section{Financial support and sponsorship \\ Nil.}

\section{Conflicts of interest}

There are no conflicts of interest.

\section{RefEREnCes}

1. American Psychiatric Association. Diagnostic and Statistical Manual of Mental Disorders (DSM-IV-TR). $4^{\text {th }}$ ed. Washington: American Psychiatric Association; 1994.

2. Meagher DJ, Trzepacz PT. Motoric subtypes of delirium. Semin Clin Neuropsychiatry 2000;5:75-85.

3. Ely EW, Inouye SK, Bernard GR, Gordon S, Francis J, May L, et al. Delirium in mechanically ventilated patients: Validity and reliability of the confusion assessment method for the Intensive Care Unit (CAM-ICU). JAMA 2001;286:2703-10.

4. McNicoll L, Pisani MA, Zhang Y, Ely EW, Siegel MD, Inouye SK, et al. Delirium in the Intensive Care Unit: Occurrence and clinical course in older patients. J Am Geriatr Soc 2003;51:591-8.

5. Inouye SK. Delirium in older persons. N Engl J Med 2006;354:1157-65.

6. Levkoff SE, Evans DA, Liptzin B, Cleary PD, Lipsitz LA, Wetle TT, et al. Delirium. The occurrence and persistence of symptoms among elderly hospitalized patients. Arch Intern Med 1992;152:334-40.

7. Lawlor PG, Gagnon B, Mancini IL, Pereira JL, Hanson J, Suarez-Almazor ME, et al. Occurrence, causes, and outcome of delirium in patients with advanced cancer: A prospective study. Arch Intern Med 2000;160:786-94.

8. Balas MC, Happ MB, Yang W, Chelluri L, Richmond T. Outcomes associated with delirium in older patients in surgical ICUs. Chest 2009;135:18-25.

9. Dubois MJ, Bergeron N, Dumont M, Dial S, Skrobik Y. Delirium in an Intensive Care Unit: A study of risk factors. Intensive Care Med 2001;27:1297-304.

10. Ouimet S, Kavanagh BP, Gottfried SB, Skrobik Y. Incidence, risk factors and consequences of ICU delirium. Intensive Care Med 2007;33:66-73.

11. Pandharipande P, Shintani A, Peterson J, Pun BT, Wilkinson GR, Dittus RS, et al. Lorazepam is an independent risk factor for transitioning to delirium in Intensive Care Unit patients. Anesthesiology 2006;104:21-6.

12. Aldemir M, Ozen S, Kara IH, Sir A, Baç B. Predisposing factors for delirium in the surgical Intensive Care Unit. Crit Care 2001;5:265-70.

13. van den Boogaard $M$, Schoonhoven $L$, van der Hoeven JG, van Achterberg T, Pickkers P. Incidence and short-term consequences of delirium in critically ill patients: A prospective observational cohort study. Int J Nurs Stud 2012;49:775-83.

14. Salluh JI, Soares M, Teles JM, Ceraso D, Raimondi N, Nava VS, et al. Delirium epidemiology in critical care (DECCA): An international study. Crit Care 2010;14:R210.

15. Ely EW, Shintani A, Truman B, Speroff T, Gordon SM, Harrell FE Jr., et al. Delirium as a predictor of mortality in mechanically ventilated patients in the Intensive Care Unit. JAMA 2004;291:1753-62.

16. Milbrandt EB, Deppen S, Harrison PL, Shintani AK, Speroff T, Stiles RA, et al. Costs associated with delirium in mechanically ventilated patients. Crit Care Med 2004;32:955-62.

17. Otter H, Martin J, Bäsell K, von Heymann C, Hein OV, Böllert P, et al. Validity and reliability of the DDS for severity of delirium in the ICU. Neurocrit Care 2005;2:150-8.

18. Ely EW, Gautam S, Margolin R, Francis J, May L, Speroff T, et al. The impact of delirium in the Intensive Care Unit on hospital length of stay. Intensive Care Med 2001;27:1892-900.

19. Ely EW, Margolin R, Francis J, May L, Truman B, Dittus R, et al. Evaluation of delirium in critically ill patients: Validation of the confusion assessment method for the Intensive Care Unit (CAM-ICU). Crit Care Med 2001;29:1370-9.

20. Lin SM, Liu CY, Wang CH, Lin HC, Huang CD, Huang PY, et al. The impact of delirium on the survival of mechanically ventilated patients. Crit Care Med 2004;32:2254-9.

21. Thomason JW, Shintani A, Peterson JF, Pun BT, Jackson JC, Ely EW, et al. Intensive Care Unit delirium is an independent predictor of longer hospital stay: A prospective analysis of 261 non-ventilated patients. Crit Care 2005;9:R375-81.

22. Micek ST, Anand NJ, Laible BR, Shannon WD, Kollef MH. Delirium as detected by the CAM-ICU predicts restraint use among mechanically ventilated medical patients. Crit Care Med 2005;33:1260-5.

23. Roberts B, Rickard CM, Rajbhandari D, Turner G, Clarke J, Hill D, et al. Multicentre study of delirium in ICU patients using a simple screening tool. Aust Crit Care 2005; 18:6, 8-9, 11-4.

24. Girard TD, Jackson JC, Pandharipande PP, Pun BT, Thompson JL, Shintani AK, et al. Delirium as a predictor of long-term cognitive impairment in survivors of critical illness. Crit Care Med 2010;38:1513-20.

25. Liptzin B, Levkoff SE. An empirical study of delirium subtypes. Br J Psychiatry 1992;161:843-5.

26. Meagher DJ, O'Hanlon D, O'Mahony E, Casey PR, Trzepacz PT. Relationship between symptoms and motoric subtype of delirium. J Neuropsychiatry Clin Neurosci 2000;12:51-6.

27. McCusker J, Cole M, Abrahamowicz M, Primeau F, Belzile E. Delirium predicts 12-month mortality. Arch Intern Med 2002;162:457-63.

28. Girard TD, Pandharipande PP, Ely EW. Delirium in the Intensive Care Unit. Crit Care 2008;12 Suppl 3:S3.

29. Shi CM, Wang DX, Chen KS, Gu XE. Incidence and risk factors of delirium in critically ill patients after non-cardiac surgery. Chin Med J (Engl) 2010;123:993-9.

30. Waldstein SR, Manuck SB, Ryan CM, Muldoon MF. Neuropsychological correlates of hypertension: Review and methodologic considerations. Psychol Bull 1991;110:451-68.

31. Madden DJ, Blumenthal JA. Slowing of memory-search performance in men with mild hypertension. Health Psychol 1989;8:131-42.

32. Pandharipande P, Cotton BA, Shintani A, Thompson J, Pun BT, Morris JA Jr., et al. Prevalence and risk factors for development of delirium in surgical and trauma Intensive Care Unit patients. J Trauma 2008;65:34-41.

33. Pandharipande PP, Pun BT, Herr DL, Maze M, Girard TD, Miller RR, et al. Effect of sedation with dexmedetomidine vs. lorazepam on acute brain dysfunction in mechanically ventilated patients: The MENDS randomized controlled trial. JAMA 2007;298:2644-53.

34. Kenna HA, Poon AW, de los Angeles CP, Koran LM. Psychiatric complications of treatment with corticosteroids: Review with case report. Psychiatry Clin Neurosci 2011;65:549-60. 
35. Fardet L, Flahault A, Kettaneh A, Tiev KP, Généreau T, Tolédano C, et al. Corticosteroid-induced clinical adverse events: Frequency, risk factors and patient's opinion. Br J Dermatol 2007;157:142-8.

36. Fardet L, Petersen I, Nazareth I. Suicidal behavior and severe neuropsychiatric disorders following glucocorticoid therapy in primary care. Am J Psychiatry 2012;169:491-7.

37. Jaber S, Chanques G, Altairac C, Sebbane M, Vergne C, Perrigault PF, et al. A prospective study of agitation in a medical-surgical ICU: Incidence, risk factors, and outcomes. Chest 2005;128:2749-57.

38. Lat I, McMillian W, Taylor S, Janzen JM, Papadopoulos S, Korth L, et al. The impact of delirium on clinical outcomes in mechanically ventilated surgical and trauma patients. Crit Care Med 2009;37:1898-905.

39. Mehta S, Cook D, Devlin JW, Skrobik Y, Meade M, Fergusson D, et al. Prevalence, risk factors, and outcomes of delirium in mechanically ventilated adults. Crit Care Med 2015;43:557-66.

40. Shehabi Y, Riker RR, Bokesch PM, Wisemandle W, Shintani A, Ely EW, et al. Delirium duration and mortality in lightly sedated, mechanically ventilated intensive care patients. Crit Care Med 2010;38:2311-8.

41. Salluh JI, Wang H, Schneider EB, Nagaraja N, Yenokyan G, Damluji A, et al. Outcome of delirium in critically ill patients: Systematic review and meta-analysis. BMJ 2015;350:h2538. 